\title{
ANALYTIC SOLUTION FOR THE PROBLEM OF GRIDDED GAP-ELECTRON FLOW INTERACTION*
}

\author{
S. Kheifets, S. Yu and J. JaEger \\ Slanford Linear Accelerator Center \\ Stanford Univeraity, Stanford, California ofs0s
}

\section{Initroduetion}

The present study is motivated by the modelling of high-power klystrons. The two basic components of a Klystron are the resonant cavitic and the drilt apaces. This puper addreses only the first of these two components. Modelling of the drift spaces is deferred to future work. The formulation is not restricted to Klystron modelling, but is applicable to any problem involving the interaction of an electron beam with a resonant cavtly.

While the theory of Klystrons has been worked out in detail in the small aignal limit, the problem remains largely unsolved when the signals are large. In particular, the hydrodynamic models of electron beans used to derive the amall signal theories fail when particle trajectories cross each other. In this paper, we employ a Vlasor description of the electron beam to study the Klystron problem. In the Vasor formulation we follow the evolution of the electron distribution function in phase-space. The general framework can naturally accommodate particle crossing, and the beam dyanmics is aceurately described even when the siguals are large.

While the Vasov formulation is equivalent in principle to a particle simulation, the mathematical structure of the Vlasov equations makes it relatively easy to build in the stendy-state condition. Since in many Klystron problems we are intorested mostly in the steady-state solution, the Vlasov deseription is very convenient. This is an advantage that a particle simulation does not share.

- Work supported by the Department of Energy, contract DE-AC03-76SF00515. 
The self-consistent solution of the gystem of Vasor equations is found under the following assumptions:

a) One dimensional (longitudinally) nonrelativistic particle flow.

b) Electric field uniform in the longitudinal coordinate (gridded gap).

The solution is valid a) for an arbitrary particle distribution of the fow entering the gap, b) (or any gap size, o) for all beam intensities, and d) for a broad class of time dependences of the electric field in the gap, although we will be studying in detail the special case of a resonant cavity with a single dominant frequency.

In section 2 the problem is formulaled in terme of the Vlasov equations. In section 3 we present the solution of the Liouville equations for the initial value problem. Then in tho next saction a boundary value solution of the biouville equation is studied. In section 5 the Maxwell equations are solved for a given current density. The results of sections $\mathbf{A}$ and 5 are combined in section 6 to produce a self-consistent solution for the Vlasov equations. In the limit of a small beam intensity and/or a small electric field the solution gives the same results as the small signal theory based on the hydrodyanmic beam models. (Section 7.) We bave also derived a genoral solution in the limit of small gap size. (Section 8.) The last sections contain a numerical example, comparison with known approximations and some conclusions as well as a discussion of possible applications of the suggested solution to the klystron problem. Some of the results presenled in this paper are not new. The derivation has been included here for completeness.

\section{The Vlasor Equations}

The most general and exact degcription of the electrommgnetic interaction of a particlo flux with eavironment is given by a system of equations describing the evolution of the particle phase space distribution function and the electromagnetic field produced by particle charges. This system of equations is referred to usually as the Vasov equations, 1,2,3 For the nonrelativistic one dimensional problem considered bere, the Vasov equations are as follows:

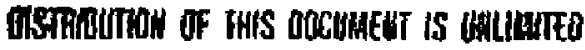

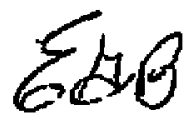


Consider the motion of an electron in the gap in a direction with the velocity $v=$ dz/dt:

$$
\frac{d v}{d \ell}=\frac{e}{m} E(t)
$$

Hore ' $E(t)$ is the z-component of the electric field assumed to depend on time only. The physical realization of such a field takes place in a gridded gap, for example.

The first two integrala of this equation aro

$$
v(t)=\frac{e}{m_{t_{0}}} \int_{t}^{t} E\left(t^{t}\right) d t+v_{0}
$$

and

$$
z(t)=z_{0}+t\left(t-t_{0}\right)-\frac{c}{m} \int_{t_{0}}^{t}\left(t-t_{0}\right) E\left(t^{\prime}\right) \mathrm{d} t
$$

The evolution of a fiux of electrons ingide the gap can be deseribed by a distribution function $\psi$ of time $t$, coordinate $z$ and velocity $v: \psi=\psi(z, v, t)$. The continuity equation in the phase spere $\pi, v$ is called the Liouville equation. In our case it looks like ( $C$ is an operator):

$$
L \psi \equiv \frac{\partial \psi}{\partial t}+v \frac{\partial \psi}{\partial z}+\frac{c}{m} E(t) \frac{\partial \psi}{\partial v}=0
$$

Notice that $v$ and $z$ in this equation are considered as independent variables.

The electric feld $E(t)$ in general can be produced by the cbarges and curreats of the fux taking into account the epvironment as well as by external sources. Introducing the vector and scales potentinls $\vec{A}(\vec{F}, t)$ and $\phi(\vec{F}, t)$ we can deseribe tho electromngnetic field by the lollowing Maxwell equations:

$$
\begin{gathered}
\frac{1}{c^{2}} \frac{\partial^{2} A}{\partial t^{2}}-\nabla^{2} A=\frac{4 \pi}{c} \vec{j}(\vec{I}, \theta) \\
\nabla \cdot \vec{A}+\frac{1}{c} \frac{\partial \phi}{\partial t}=0
\end{gathered}
$$




$$
\vec{E}=-\nabla_{\phi}-\frac{1}{c} \frac{\partial \vec{A}}{\partial t}
$$

The current density $\vec{j}(\vec{F}, t)$ in $(2.5)$ in turn can be expressed as a sum of external current density $\vec{f}_{e x t}$ (produced for example by an external RF generator) and the current density of the fux itself. In our case, we assume for simplicity that the electron current is concentrated on axis, and write

$$
\begin{aligned}
& f(\vec{r}, t)=\boldsymbol{J}_{\mathrm{ext}}+\frac{\delta(r)}{2 \pi r} J(z, t), \\
& I(z, l)=\varepsilon \int_{-\infty}^{\infty} d v \cdot v \psi(z, v, l)
\end{aligned}
$$

The system of equations (2.4) through (2.9) are the Vlasov equations. The solution of this system satisfying all the necessary initinl and bnundary conditions is the self consistent solution of the problem. The search lor such a solution is the subject of the present paper.

\section{Initial Value Solution Or The Llouville Equation}

We solve first the Louville equation (2.4) agguming for the time being $E(t)$ as a given function of time. It is known, that any lunetion of the integrals of motion is the solution of the Liouville equation. Hence the function

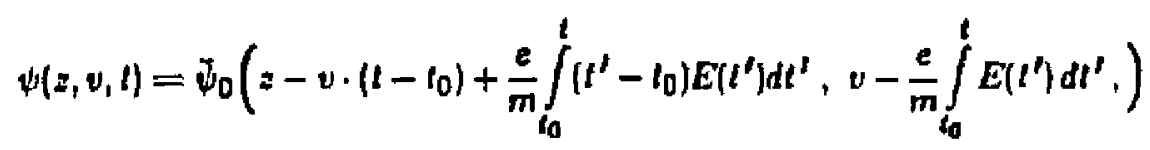

is a solution of equation $(2.4)$. $\mho_{0}(z, v)$ corresponds to the initial distribution at $t=$ $\iota_{0}$. It is easy to check by direct substitution, that (3.1) indeed satisfies (2.11). We will not do this here since we are interested in the solution of the boundary value probjem rather than the initial value problem. 


\section{Boundary Value Solution Or The Llonvllle Equation}

Suppose now that at $z=z_{0}$ the distribution function is given for all times and velocities:

$$
\psi_{0}=\psi_{0}(v, t)
$$

We are intarested now in fading a solution $\psi(z-z 0, v, l)$ of $(2.4)$ which goes into (4.1) for $z \rightarrow z_{0}$. This solution will describe the evolution of $\psi_{0}$ in $z_{1}$ and $l$. In particular, it will give us the distribution function $\psi(d, v, t)$ at the exit of the gap $z=z_{0}+d$.

The aim is achieved in the following way. Introduce first the implicit function $\theta\left(z-z_{0}, v, t\right)$ as a solution of the equation

$$
r\left(z-z_{0}, v_{1}, \theta\right) \equiv z-z_{0}-v \cdot(t-\theta)+\frac{\epsilon}{m} \int_{\theta}^{t}\left(t^{\prime}-\theta\right) E(t) d t=0
$$

which satisfies the condition:

$$
\theta(0, v, t)=t
$$

Introduce next the function

$$
V\left(z-z_{0}, v, 0\right)=v-\frac{e}{m} \int_{\theta\left(x-z_{0,0,0}\right)}^{t} E\left(t^{\prime}\right) d r^{\prime}
$$

From (4.3) it follows immediately

$$
V(0, v, l)=v
$$

Then

$$
\psi(z, v, 0)=\psi_{0}\left(V\left(z-z_{0}, v, t\right), \quad \theta\left(z-z_{0}, v, t\right)\right)
$$

is such a solution of the Liouville equation $(2,4)$ which goes into the buundary value (4.1) when $z \rightarrow z$. To prove this, note first of all tbat

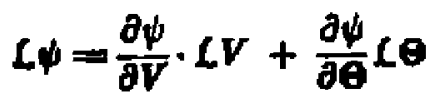


Then it is easy to see that

$$
L V=\frac{e}{m} E(\theta) \cdot \mathcal{L} \theta
$$

therefore the only thing we have to slow lo that

$$
c \theta=0
$$

Indeed, if (4.7) is true then $\mathcal{L} \psi=0$ and $(4.3)$ and $(4.5)$ provide that $\psi l_{z=0}=$ $\psi_{0}(\nu, 1)$. To prove $(4,7)$ find $L F$ (which is 0 since $F=0$ )

$$
L F=V \cdot L \theta=0
$$

Hence (4.7) is true. In the particular cese of a barmonic electrieal field:

$$
E_{h}(t)=E_{0} \cos (\omega t+\varphi)
$$

Jormula (4.4) gives:

$$
V_{h}=t-\frac{e E_{0}}{m \omega}\left[\sin (\omega t+\varphi)-\operatorname{ain}\left(\omega \theta_{h}+\varphi\right)\right]
$$

$\theta_{h}$ satialies the following equation (from formula (4.2)):

$$
\begin{aligned}
z-z_{0}-v\left(t-\theta_{h}\right) & +\frac{c E_{0}}{m \omega^{2}}\left[\cos (\omega t+\varphi)-\cos \left(\omega \theta_{h}+\varphi\right)\right] \\
& +\frac{e E_{0}}{m \omega}\left(t-\theta_{h}\right) \sin (\omega t+\varphi)=0
\end{aligned}
$$

The solution (1.6) possesses an important feature of periodicity. Nhmely, if $\psi_{0}(v, t)$ and $E(t)$ are both periodic in time ( $T$ is the period):

$$
\begin{gathered}
\psi_{0}(v, t+T)=\psi_{0}(v, t) \\
E(t+T)=E(t)
\end{gathered}
$$


then

$$
\begin{aligned}
\psi\left(z, v_{1} t+T\right) & =\psi_{0}(V(t+T), \Theta(t+T)+T) \\
& =\psi_{0}(V(t), \theta(t))=\psi(z, v, t),
\end{aligned}
$$

i.e. it is also periodic.

The correctness of this statement is very easy to see in the simple case of the harmonic electrical feld with $w=2 \pi / T$. In this case (410) is invariant under trangformation $t \rightarrow \ell+T, \theta \rightarrow \theta+T$ and so is (4.0). The proof for more general periodic function

$$
E(t)=\sum_{n} E_{n} \cos \left(n \omega t+\varphi_{n}\right)
$$

is more elaborate and wo will not give it here.

The constants $E_{n}, \varphi_{n}$ sre to be found self-conaistently from the solution of the Maxwell equation with the current deasity as a source of the fleld defined in (2.8) and (2.9).

\section{Solution Of The Maxwell Equatione}

In this seetion we consider the eurrent density a a given quantity. The solution of the Maxwell equation for an arbiltary cavity is well known and is given here only for reader's convenience.

Any cavity with the volume $\Omega$ and the metallic internal surface $\Sigma$ can be characterized by a set of ito eigen vector-potential funetions $A_{n}(F)$ satisfying the following system of uniform equations

$$
\nabla^{2} A_{n}+k_{n}^{2} \vec{A}_{n}=0
$$

and the houndary conditions

$$
\left.\vec{A}_{n}\right|_{\operatorname{lan} n x}=0
$$

The eigenvalues $k_{n}$ are delined by the cavity geometry. 
The eigenfunctions $\vec{A}_{n}$ corresponding to different field modes are orthogonal to ach other. Since (5.1) and (5.2) are linear and uniform, the eigenfunctions can be multiplied by an asbitrary factor. It is convenient to normalize them in the following way

$$
\int_{\Omega} \lambda_{n} \cdot \lambda_{m} d^{3} r=\delta_{n m},
$$

where $\hat{\phi}_{n m}$ is the Kronecker symbol

$$
\delta_{n m}= \begin{cases}1 & m=n \\ 0 & m \neq n\end{cases}
$$

Given the current density, the olectromagnetic field of the cavity can be found in terms of the eigenfunetions $\vec{A}_{n}(\vec{r})$. Let us represent first the vector potential $\vec{A}(\vec{r}, n)$ as an expansion in the eigenfunctions $\bar{A}_{n}$ :

$$
\vec{A}(\vec{\nabla}, l)=\sum_{n} a_{n}(t) \vec{A}_{n}(\vec{\nabla})
$$

Hore $a_{n}(t)$ are time dupendent mode "nmplitudes". Due to (5.2) this function also satisfles the boundary conditions. Substitute now (6.5) into $(2.5)$, multiply by $\vec{A}_{m}$ and integrate over the cavity volume. Using (5.3) one gets separate equation for each of the amplitudes $a_{n}$ :

$$
\frac{d^{2} a_{n}}{d l^{2}}+w_{n}^{2} a_{n}=4 \pi c \int_{\Omega} d^{3} r \vec{j} \cdot \lambda_{n}
$$

where $\omega_{n}=k_{n} \cdot c$.

The field description presented here does not include the energy dissipation in the cavity walls. There are several ways to take into account losses. One ellective way to do that is to modify (5.6) slightly:

$$
\frac{d^{2} a_{n}}{d l^{2}}+2 \alpha_{n} \frac{d a_{n}}{d t}+\omega_{n}^{n} a_{n}=4 \pi c \int d^{3} r \vec{j} \cdot A_{n} \quad
$$

where $\alpha_{n}$ is a constant describing the rale of the deray of the nlk ninde due to the energy loss (including ohmic loss). 
Solution of (5.7) is easy to obtain by the Fourier transformation of both sidea. For any function $f$ perlodic in time with period $T=2 \pi / \omega$;

$$
\begin{aligned}
& f(t)=\sum_{m} f_{m} e^{+i m w t} \\
& f_{m}=\frac{1}{T} \int_{0}^{T} d t e^{-i \omega m t} f(t)
\end{aligned}
$$

We are not interested in transient processes here. Then, for the equilibrium solution of (5.7) we get

$$
a_{n m}=\frac{4 \pi c J_{n} d^{3} r j_{m} \cdot A_{n}}{w \frac{n}{n}-m^{2} \omega^{2}+2 i \alpha_{n} m_{m}}
$$

where $\jmath_{m}$ is the $m$ th Fourier componant of the current density. For the $m$ th harmonic of the vector potential $\boldsymbol{\lambda}_{m}$ one finds from (5.5):

$$
A_{m}(\vec{\imath})=\sum_{n} \frac{4 \pi c A_{n}(\vec{r}) \int_{n} d^{3} r j_{m} \lambda_{n}}{w_{n}^{2}-m^{2} \omega^{2}+2 i \alpha_{n} m \omega}
$$

C- Thn integrals on the right hand, gide of this expression are the coupling coefficients of the mth currest harmonic to the nth cavity mode.

The mth harmonic of the eloctrical field $\vec{E}_{m}(\vec{\nabla})$ can be found from $\vec{A}_{m}$ (ㄱ):

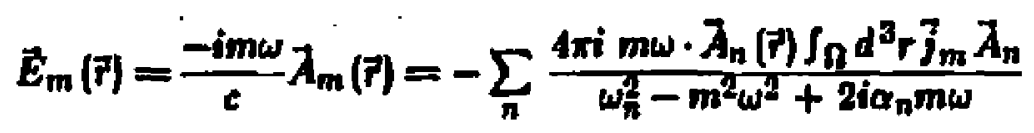

As the simplest example of the general formulae (5.11) and (5.12) let us consider a pillbox cavity with the radius $R$ and length $d$. For cylindrically symmetric current Glowing on the cavity axis the azimutbal eigennumber is equal zero. In this case the vector potential has only one nontrivinl longitudinal component $A_{n}$. Since we restrict ourselves to fields uniform in $z$ only, the longitudinal eigennumber is also equal to zero. The radial eigenaumber $n$ is related to different radial modes:

$$
A_{n}=B_{n} J_{0}\left(\gamma_{n} r / R\right)
$$


where $\gamma_{n}$ is $n t h$ root of the zeroth order Besgel funetion $J_{0}(x)$. The pormalization constants $D_{n}$ are;

$$
B_{n}=\frac{1}{\sqrt{\pi d} R \cdot J_{1}\left(\gamma_{\pi}\right)}
$$

The eigentrequencies $\omega_{n}$ in this cnse are defined by

$$
\omega_{n}=\tau_{n} c / R
$$

with the first three values of $\gamma_{n}$ being $2.405,5.520$ and 8.054 . Let us now nssume for the current density the uniform dependence on the radial distance from the axis in the interval $0<r<b$ :

$$
\begin{gathered}
j(z, r, t)=g(r) l(z, l) \\
g(r)= \begin{cases}1 / \pi b^{2} & r<b \\
0 & r \geq 0\end{cases}
\end{gathered}
$$

Then, from (5.12) we get for the longitudinal component of the elertric field:

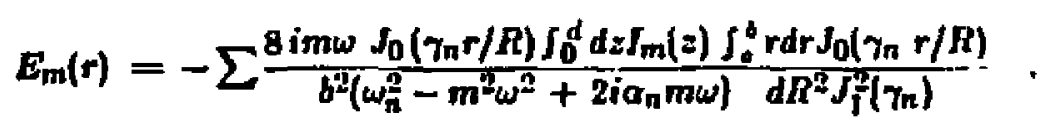

where

$$
I_{m}(z)=\frac{\omega}{2 \pi} \int_{0}^{2 \pi / \omega} d t e^{-i m \omega t} /(z, t)
$$

is the $\mathrm{m} t h$ Fourier harmonic of the current $I(z, 1)$. Define now the gap voltage harunnie

$$
V_{m}=-d\left(E_{m}(r)\right)
$$

where $\left\langle E_{m}(r)\right\rangle$ is the average electric field over the heam roses section $\left(E_{m}(r)\right)=\frac{1}{\pi} \int_{0}^{b} \operatorname{rd} d \varphi E_{m}(r)$ and the gap impedatice

$$
Z_{m}=\frac{V_{m}}{\left(S_{m}\right)}
$$




$$
\left(I_{m}\right)=\frac{1}{d} \int_{0}^{d} d z I_{m}(z)
$$

For the gap impedance $Z_{m}$ we get from (5.18)

$$
\because, z_{m}=\sum_{n} \frac{\because 16 \text { imwd } J_{j}^{2}\left(\gamma_{n} b / R\right)}{\tau_{n}^{2} J_{i}^{2}\left(\gamma_{n}\right)\left(\omega_{m}^{2}-m^{2} \omega^{2}+2 i \alpha_{n} m \omega\right) b^{2}}
$$

In a particular case when mw is close to ope of the eigenfrequenciu $\omega_{n}$, only one mode contributes to $Z_{m}$. In such a case:

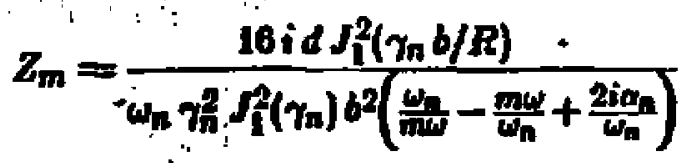

$\therefore$ or substituting $\omega_{n} / m \omega=f_{0} / /$ and Jelining $Q=\omega_{0} / 2 \alpha_{0}$

$$
Z_{m}=\frac{8 d J_{l}^{2}\left(\gamma_{n} b / R\right) Q}{\pi / n \gamma_{n}^{2} J_{l}^{2}\left(\gamma_{n}\right) b^{2}\left[1+i Q\left(\frac{1}{f_{0}}-\frac{b}{f}\right)\right]}
$$

If one represents $Z_{m}$ in the form

$$
z_{m}=\frac{(R / Q)_{h h} \cdot Q}{1+i Q\left(f_{n}-4\right)}
$$

then

$$
(R / Q)_{a h}=\frac{8 d J_{(1}^{2}\left(\gamma_{n} b / R\right)}{\pi f_{n} \gamma_{n}^{2} J_{1}^{2}\left(\gamma_{n}\right) b^{2}}
$$

For $6 \rightarrow 0$

$$
(R / Q)_{a h} \rightarrow(R / Q)_{b \rightarrow 0}=\frac{2 d}{\pi / n R^{2} J_{1}^{2}\left(\gamma_{n}\right)}
$$

The dependence of $(R / Q)_{\text {ah }}$ on the eavity length $d$ is contained solely in the factor $(R / Q)_{1,0}$. Rewrite (5.26) in the form

$$
(R / Q)_{\mathrm{A} h}=(R / Q)_{\Delta \|_{0}} \cdot m^{2}(6 / R)
$$

where $(R / Q)_{a A_{0}}$ is defined in $(5.27)$ and $m(x)=2 J_{1}(x) / x$

Congider a numerical example of a cavity with the dimensions $R=1.43 \mathrm{~cm}, \mathrm{~d}=$ $0.5 \mathrm{~cm}$ with B ground mode $(\gamma=2.405)$ on the firequency $f=2.856 \mathrm{GHz}$. From (5.27) one gets $(R / Q)_{\omega_{0}}=180 \mathrm{ohm}$. 
Depeadence of $m^{2}(x)$ is jllustrated in Table 5.1

TABLE 5.1

\begin{tabular}{|c|c|c|}
\hline$x$ & $m(x)$ & $m^{2}(x)$ \\
\hline 0.0 & 1.00 & 1.00 \\
\hline 0.2 & 0.97 & 0.04 \\
\hline 0.4 & 0.89 & 0.79 \\
\hline 0.6 & 0.76 & 0.58 \\
\hline 0.8 & 0.60 & 0.36 \\
\hline 1.0 & 0.43 & 0.18 \\
\hline
\end{tabular}

For the uniform beam with the radius $b=1.0 \mathrm{~cm}$ we get $(R / Q)_{a h}=80 \mathrm{ohm}$.

\section{Self-consistent Solution Or The Vlanor Equations}

Introducing the notion of the impredance $Z_{m}$ we can rewrite (5.21) in the following way:

$$
E_{m}=-\frac{Z_{m}}{d^{2}} \int_{0}^{d} d z I_{m}(z),
$$

where $E_{m}=-V_{m} / d$ and $I_{m}$ are the $m$ th harmonics of the electric field and of the full current flowing through the gap, respectively. It is more convenient lo consider the current due to electron flow separately from other possible currents, e.g. the eurrent arising trom the external generator.

Consider for example the first klystron cavity. Assume that the cold eavity is exited by an external rf generator. Then it is convenient to rowrite (6.1) in the following form:

$$
E_{m}=E_{m e x t}-\frac{Z_{m}}{d^{2}} \int_{0}^{d} d z I_{m}(z)
$$

where now $I_{m}$ is the $m l h$ hurmonic of the electron flow current which in turn depends on $E_{m}, E_{m \text { ert }}$ is the mth harmonic of the feld excited in the cold eavity by an external generator. It can be equal zero in particular case of oot excited cavity (for example the second klystron cavity). 
Suppose now that resonance denominators in (5.18) are small for modes which result in the uniform feld distribution along the longitudinal coordinate $z$ and large for all other modes: One guch an example provides the pilibox cavity excited on the ground mode. The gridded gap might be another, example. In such a cage the electric Geld is fully determined by its harmonic ampitudes $] E_{m}$ ] and phases arg $\left(E_{m}\right)$ of [6.2). The integral of the right hand side of (6.2) in turn depends on electric field through the current tarmonic (5.10).

Consider the simplest case when only one mode (say the zeroth one) and one harmonit (assume the fist one) contributes to the sum (5.18). Then (6.2) constilutes two traoscendental equations for the amplituda $E_{0}$ and the phase $\varphi$ (or for the real and imaginary parts) of the first harmonic of the feld. Solution of these equations provide the self-consistent field $E_{h}=E_{0} \cos (w t+\varphi)(4.8)$. Substitute this field back into the solution (4.6) for the distribution function. One gets now the selfconsistent solution of the Vasov equation which satisfie the boundary value st $z=z_{0}$.

As an example, let os assume for the initial electron fow a de current with no velocity spread:

$$
\left.\psi_{0}(v, l)\right|_{a \rightarrow s_{0}}=\frac{L_{0}}{c t_{0}} d\left(v-v_{0}\right),
$$

where $I_{0}$ in the de electron current, and $v_{0}=\sqrt{2 \mathrm{eV} / \mathrm{m}}$ is the initial velocity due to the de gun voltage $V_{0}$. At this point it is convenient to introduce the following dimensioaless variables

$$
\begin{array}{ll}
x=\left(z-z_{0}\right) / d & 0 \leq x \leq 1 \\
u=v / \omega d & -\infty \leq u \leq \infty \\
r=\omega t+\varphi & \\
\tau_{0}=\omega \theta+\varphi \\
k=e E_{0} / \omega+s^{2} d
\end{array}
$$


According to (4.0) the distribution function for any later coordinate and time is in the new varisbles

$$
\psi(x, u, \tau)=\frac{I_{0}}{c_{0}} \delta\left(u-k \operatorname{ain} r+k \operatorname{ain} \tau_{0}-u_{0}\right),
$$

where $u_{0}=v_{0} /$ wd and the function $\tau_{0}=\tau_{0}(x, t, \tau)$ is defined by squation:

$$
x-u\left(r-\tau_{0}\right)+k \mid \cos r-\cos \tau_{0}+\left(r-\tau_{0}|\sin r|=0\right.
$$

From the distribution function $(6,9)$ one can find the beam density current

$$
\begin{aligned}
I(x, \tau) & =\frac{I_{0}}{u_{0}} \int_{-\infty}^{\infty} d u u \delta\left(u-u \sin \tau+u \sin \pi-u_{0}\right) \\
& =I_{0} \frac{u(\tau)}{\left.\mid u_{0}+k \| \tau-\tau_{0}(\bar{u})\right] \cos \tau_{0}(\overline{0}) \mid}
\end{aligned}
$$

where $n=a(\tau)$ is the solution of equation

$$
\theta+k \sin r_{0}(t)=u_{0}+k \sin T
$$

The first hnrmonic of $I(x, t)$ is

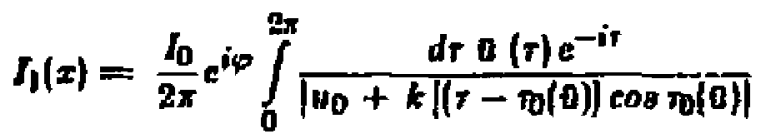

Calculate now the average ovur $x$ of this current and substitule into (6.2). Nole that $E_{1}=E_{0} e^{i p} / 2$ and $E_{1 e x t}=E_{D e x l} / 2$.

$$
k=k_{t x l} e^{-i \varphi}-\frac{2 Z_{1}}{d^{2}} \frac{e I_{0}}{2 \pi m \omega^{2}} \int_{0}^{1} d x \int_{0}^{2 \pi} \frac{d t a(t) e^{-i \tau}}{\left|t_{0}+k\left[\left(\tau-\tau_{0}\right)\right] \cos \tau_{0}\right|},
$$

where $k_{\text {trt }}=e E_{0 \text { ext }} / m \omega^{2} d$. The complex equation (6.14) is equivalent to two transcendental equations which define the amplitude $E_{0}$ and the phose $\varphi$ fin respect to the external field) of the field in the gap.

Below we provide numerical rwults, which illustrate the application of the derived formulae. 


\section{$\therefore$ 7. Small Slgal Approximation}

It is instructive to atudy the obtained results in the limit of a small electric fieid and to compare them with the known results from the amall signal theory.

In the small signal limit the lowest power of the parameter $k$ should be retsined in all expangions in power seria.

In variables (6.4) - (6.8), equations (4.0), (4.10) for $n=V_{h} / \omega d$ and $m$ look like:

$$
\begin{gathered}
0=u-k \sin \tau+k \sin t_{0} \\
x-u\left(\tau-\tau_{0}\right)+k\left(\cos \tau-\cos \tau_{0}+\tau \sin \tau-\tau_{0} \sin \tau\right)=0
\end{gathered}
$$

The solutions of (7.1) ond (7.2) to the first order in $k$ are

$$
\begin{gathered}
u=u-k \sin r+k \sin \left(r-\frac{x}{u}\right) \\
\tau_{0}=\tau-\frac{x}{u}+\frac{k}{u}\left[\cos \left(\tau-\frac{x}{u}\right)-\cos \tau-\frac{t}{u} \sin t\right]
\end{gathered}
$$

The terms independent of $k$ here give the balliatic approximation. The last terms in (7.3) and (7.4) represent the influence of the electric field.

Let us assume for simplicity that the distribution function of the elevtron flow on the entrance of the gap is (B.3).

\subsection{Couphing CoEfFicient}

Let us firgt of All find the expression for the coupling coefficient $\mu$ as it follows from our solution. One can define $\mu$ as the ratio of the average kinetic energy change to the maximum of the energy gain in the gap. Caleulate arst the avernge $\left(u^{2}\right)$ as the function of $x$ :

$$
\left(u^{2}\right)=\frac{\int_{-\infty}^{\infty} d u u^{2} \delta\left[u-k \sin t+k \sin \left(r-\frac{x}{u}\right)-u_{0}\right]}{\int_{-\infty}^{\infty} d u \delta\left[u-k \sin r+k \sin \left(r-\frac{x}{u}\right)-u_{0}\right]}
$$


To the first order in $k$ for $x=1$

$$
\left(v^{2} h_{1}=v_{0}^{2}+2 k u_{0}\left[\sin r-\sin \left(r-\frac{1}{u_{0}}\right)\right]\right.
$$

Froin bere we get $\left(\phi\right.$ denotes $\left.\tau+1 / 2 u_{0}\right)$

$$
\mu=\frac{\left(u^{2}\right)_{1}-u_{0}^{2}}{2 k \cos \phi}=\frac{\sin \theta}{\theta},
$$

where $\theta=\frac{1}{x+\frac{w d}{2}}=\frac{w d}{2 w_{0}}$ is the half of the gap transit angle.

\subsection{Beam Loading}

Now, let us consider the beam londing by the alectron flow as it follaws fiom our solution. We need now the expression for the bean current density in the small signal approximation. The charge and the current densities both can found by integration of (0.9)

$$
\begin{aligned}
& \rho(x, \tau)=\frac{J_{0}}{v_{0} w d} \int_{-\infty}^{\infty} d u \delta\left[u-k \sin \tau+k \sin \left(\tau-\frac{x}{u}\right)-u_{0}\right] \\
& I(x, \tau)=\frac{J_{0}}{u_{0}} \int_{-\infty}^{\infty} d u u \delta\left[u-k \sin \tau+k \sin \left(\tau-\frac{x}{v}\right)-u_{0}\right]
\end{aligned}
$$

Pertorming the intugrations we find to the first order in $k$ :

$$
\begin{aligned}
& J(x, \tau)=I_{0}\left\{1+\frac{k}{\tau_{0}}\left[\sin \tau-\sin \left(\tau-\frac{\tau}{u_{0}}\right)\right]-\frac{x k}{u_{0}^{2}} \cos \left(\tau-\frac{x}{u_{0}}\right)\right\} \\
& \rho(x, \tau)=\frac{I_{0}}{u_{0} w_{d}}\left(1-\frac{k x}{u_{0}^{2}} \cos \left(\tau-\frac{x}{u_{0}}\right)\right)
\end{aligned}
$$

It is asy to sea that (7.10) and (7.11) salisly the continuity equation

$$
\frac{\partial \rho}{\partial r}+\frac{1}{w d} \frac{\partial I}{\partial x}=0
$$

As one sees from (7.10) in the small signal approximntion the current density besitles the de component contains anly the first harmonic. Expression (7.10) can be obtained conversely by expanding expression (6.11) in the power series in parameter $k$. 
Using the definition (5.10) the first harmonic of the bean current density is

$$
I_{1}(x)=I_{0} e^{i \varphi} \frac{h}{2 u_{0}}\left(\sin \frac{x}{u_{0}}-\frac{x}{u_{0}} \cos \frac{x}{u_{0}}-i+i \cos \frac{x}{u_{0}}+i \frac{x}{u_{0}} \sin \frac{x}{u_{0}}\right)
$$

This expression is the asme as one obtained from formula (2.1) of the paper" assuminr; $\dot{E}_{x}=$ conot. It also coincides with the corresponding expression for iv in the book."

Let us rewrite equation (6.2) in variables (6.4) - (6.7):

$$
\theta_{1}=\frac{Z_{1}}{d}\left[I_{1 \text { ext }}-\int_{0}^{1} d x I_{1}(x)\right]
$$

Here $I_{\text {lext }}$ is the first harmonic of the external current excitiog the cavity. Substitute now (7.13) into (7.14) and take into account that $E_{1}=E_{0} e^{i p} / 2, I_{\text {lext }}=I_{0 e x t} / 2$. Then:

$$
E_{0}=\frac{Z_{1}}{d} I_{0 \mathrm{ext}} \mathrm{e}^{-i p}-\frac{Z_{1}}{d} I_{0} k(B+i A)
$$

where

$$
\begin{gathered}
B=\int_{0}^{1 / t_{0}} d \sigma(\sin \theta-\sigma \cos \theta)=4 \theta \sin \theta\left(\frac{\sin \theta}{\theta}-\cos \theta\right), \\
A=-\int_{0}^{1 / t_{0}} d \sigma(1-\cos \sigma-\sigma \sin \theta)=\cdot \theta \cos \theta\left(\frac{\sin \theta}{\theta}-\cos \theta\right),
\end{gathered}
$$

Here $\theta=1 / 2 u_{0}=w d / 2 v_{0}$ is balf of the transit angle for the gap. Solving (7.15) in respect to $E_{0}$ one finds:

$$
E_{0}=\frac{J_{0 e x t} e^{-i p}}{d\left[\frac{1}{Z_{t}}+\frac{d_{0}(B+i A)}{m \omega^{2} d^{2}}\right]}
$$

Substitute now expression (5.24) for $Z_{1}$ :

$$
E_{0}=\frac{(R / Q)_{s h} I_{0 e z t} e^{-i \varphi}}{\left.d \frac{f}{d}+i\left(\frac{f}{f_{0}}-\frac{h}{f}\right)+\frac{D_{0}(A / Q)_{h+}(B+i A)}{2 V_{0}}\right]}
$$


From formula (7.19) immediately follow the usual expressions for the londed quality $Q_{L}$ and the shifted frequency $f_{L}$ of the gap:

$$
\begin{aligned}
& \frac{1}{Q_{L}}=\frac{1}{Q}+\frac{I_{0}(R / Q)_{\Delta h}}{2 V_{0}} \frac{\sin \theta}{\theta}\left(\frac{\sin \theta}{\theta}-\cos \theta\right) \\
& f_{i}=f_{0}\left[1-\frac{I_{0}(R / Q)_{\Delta h}}{4 V_{0}} \frac{\cos \theta}{\theta}\left(\frac{\sin \theta}{\theta}-\cos \theta\right)\right] \\
& \text { B. Narrow Gap Approximation }
\end{aligned}
$$

\section{B. Narrow Gap Approximation}

In this section, we derive an analytic solution to the Vlasov equation in the limit of narrow gnps. The expansion is not restricted to small signals, but the regult is consistent with small signal theory in the proper limit.

The assumption of narrow gaps allows us to expand the distribution fuaction in a Taylor series. We have in general

$$
\psi(z)=\left.\sum_{n=0}^{\infty} \frac{1}{n !} \frac{\partial^{n} \psi}{\partial z^{n}}\right|_{=_{0}}(z-z 0)^{n}
$$

,For narrow gaps, the solution is given by the Hrst tew terms of the serito. We will work out the example of an initial cold distribution, with

$$
\psi_{0}(v, t)=\frac{J_{0}}{e v_{0}} \delta\left(v-v_{0}\right)
$$

the geveral solution is given by

$$
\psi(z, v, t)=\frac{t_{0}}{e t_{0}} \delta\left(v-\int_{\theta}^{t} a\left(t^{\prime}\right) d t^{\prime}-v_{0}\right)
$$

where $\theta$ is given by

$$
z-z_{0}=v(t-\theta)-\int_{\theta}^{t}\left(t^{\prime}-\theta\right) a\left(t^{\prime}\right) d t^{\prime}
$$


We will perform the expansion of (8.3) up to $n=3$. The frst term in the series $(n=0)$ is given of course by pot $(v, i)$ as defined in Eq. (8.2). To obtain the $n=1$ term, we need to evaluate

$$
\therefore \frac{\partial \psi}{\partial z}=\frac{\partial \psi_{0}}{\partial \theta} \sigma(\theta) \frac{\partial \theta}{\partial z}
$$

Differentiating Eq. (8.4), we obtain

$$
\frac{\partial z}{\partial \theta}=-v+\int_{\theta}^{t} a\left(t^{\prime}\right) d t^{\prime}
$$

The coefficient of the $n=1$ term is then given by

$$
\left.\frac{\partial \psi}{\partial z}\right|_{\partial_{0}}=-\frac{I_{0}}{e \varepsilon_{0}} \delta^{\prime}\left(v-v_{0}\right) a(t) / v, n=1
$$

The coeffieient of $n=2 \mathrm{term}$ is proportionsl to the second derivative of $\phi$ and is evaluated to be

$$
\left.\left(\frac{e v_{0}}{I_{0}}\right) \frac{\partial^{2} \psi}{\partial z^{2}}\right|_{z_{0}}=\delta^{\prime \prime}\left(v-\omega_{0}\right) \frac{a^{2}(t)}{v^{2}}+\delta^{\prime \prime}\left(v-v_{0}\right)\left[\frac{a^{\prime}(t)}{v^{2}}-\frac{a^{2}(t)}{v^{3}}\right], n=2
$$

Note that $\delta^{\prime}$ refers to the derivative of the delta function with respect to velocity while $\alpha^{\prime}(t)$ is a derivative of the scceleration with respect to time. A superscript with $n$ primes refers to the $n$th derivative. Finally, the $n=3$ coeficient is evaluated to be

$$
\begin{aligned}
\left.\left(\frac{e v_{0}}{I_{0}}\right) \frac{\partial^{3} \psi}{\partial z^{3}}\right|_{z_{0}} & =-\delta^{m \prime}\left(v-v_{0}\right) \frac{a^{3}}{v^{3}}+3 \delta^{\prime \prime}\left(v-v_{0}\right)\left[\frac{a a^{\prime}}{v^{3}}+\frac{a^{3}}{v^{3}}\right] \\
& -\delta^{\prime}\left(v-v_{v}\right)\left[\frac{a^{\prime \prime}}{v^{3}}-\frac{1 a^{\prime} a}{v^{4}}+\frac{3 a^{3}}{v^{5}}\right], n=3
\end{aligned}
$$

The current is related to the first moment of the distribution function

$$
\lambda(z, t)=e \int d v v \psi(z, v, t)
$$

In the Taylor series expansion of $\psi$, the velocity integrals may be evaluated term by term. The $n=0$ term gives rige to the d.c. component of the cursent since

$$
c \int d v v \psi_{0}=\frac{J_{0}}{v_{0}} \int d v v \tilde{\omega}\left(v-v_{0}\right)=I_{0}
$$


The $n=1$ component gives no contribution since

$$
\left.e \int d v v \frac{\partial \psi_{0}}{\partial z}\right|_{\varepsilon_{0}}=-\frac{J_{0}}{\nu_{0}} a(t) \int d v \delta\left(v-\nu_{0}\right)=0
$$

The $n=2$ component of the distribution function has terms which are proportional to $a^{7}(t)$. Hawever, these two terms cancel exactly when we tale the velocity moment of the distribution function. We are then left with a contribution to the if current

$$
\text { e }\left.\int d v v \frac{1}{2} \frac{\partial^{2} \psi_{0}}{\partial z^{2}}\right|_{\sigma_{0}}\left(z-z_{0}\right)^{2}=\frac{I_{0}}{2 v_{0}^{3}} a^{\prime}(t)\left(z-z_{0}\right)^{2}
$$

To evoluate the $n=3$ component of the current, we take the velocity moment of Eq. (8.0). Again, the terms proportional to $a^{3}(t)$ vapish and we obtain

$$
\left.\int d v v \frac{1}{B} \frac{\partial^{3} \psi_{0}}{\partial s^{3}}\right|_{\delta_{0}}\left(z-z_{0}\right)^{3}=I_{0}\left(\frac{5 a a^{\prime}}{v_{0}^{5}}-\frac{a^{\prime \prime}}{3 v_{0}^{4}}\right)\left(z-z_{0}\right)^{3}
$$

In performing the velocity integrals, we have made use of the delta function identity

$$
\int g(v) \delta^{(n)}\left(v-v_{0}\right) d v=\left.(-1)^{n} \frac{\partial^{n} g}{\partial v^{n}}\right|_{t_{0}}
$$

Combining these results, we have that to $n=3$ in the Toylor series expansion,

$$
I(z, t)=I_{0}\left\{1+\frac{a^{\prime}(t)}{2 v_{0}^{3}}\left(z-z_{0}\right)^{2}-\left[\frac{a^{\prime \prime}}{3 v_{0}^{\prime}}-\frac{5 a a^{\prime}}{v_{0}^{5}}\right]\left(z-s_{0}\right)^{3}\right\}
$$

The torm which is proportional to $a a^{\prime}$ reprogents our Grst explicit nonlinear contribution to the current. Howaver, it is clear that if $a(l)$ is a pure first barmonic, the quadratic term in a can contribute only to the zeroth and second barmonic. Hence, to the order considered, there is no higher order contribution to the if component of the current.

We now turn to examine more carefully the z-dependence of $J_{1}$. The first harmonis of the current is related only to the linear tertas in a(l).

For

$$
a(t)=\frac{e v}{m d} \cos (w t+p)
$$


we have

$$
\begin{aligned}
& I_{1}\left(z_{1} l\right)=\frac{b_{0}}{2 v_{0}^{3}}\left[a^{\prime}(\theta)\left(z-z_{0}\right)^{2}-\frac{2 a^{\prime \prime}(t)}{3 v_{0}}\left(z-z_{0}\right)^{3}\right] \\
& =-\frac{I_{0} \omega}{2 v_{0}^{3}} \frac{e V}{m d}\left(z-z_{0}\right)^{2}\left[\sin (\omega t+\varphi)-\frac{2 \omega\left(z-z_{0}\right)}{3 \omega_{0}} \cos (\omega t+\varphi)\right] \\
& \approx-\frac{L_{0} \omega}{2 v_{0}^{3}} \frac{c V}{m d}\left(x_{1}-z_{0}\right)^{2} \sin \left(\omega t+\varphi-\frac{2 \omega\left(z-z_{0}\right)}{3 \omega_{0}}\right) \\
& \therefore=\frac{I_{0} \omega}{2 \varepsilon_{0}^{3}} \frac{e V}{m d}\left(z-z_{0}\right)^{2} \cos \left(\omega t+\varphi+\frac{\pi}{2}-\frac{2 \omega\left(z-z_{0}\right)}{3 \omega_{0}}\right)
\end{aligned}
$$

The analytic formula prediets a quadratic $z$-dependence on the amplitude and a linear $z$-depeadence on the phase of $\dot{l}_{1}$.' These fentures are consistent with the numerical results from Section 9. The magnitude of the amplitude and rate of phase change are also in agreemenit.

\section{Numerical Reanlita Comparioon With The Small Signal Approx}

Here we apply formulae to the input klyatron cavity. In general, the small signal approximation gives correct results for that eavity. This is true due to small volues of both the input power and the Iength of the gap. In addition, the initial distribution "of electrons at the cavity entrance in velocities and time is very simple and is ensy to simulate. Hence, in this case the results obtained by using the self-consistent solution should agree with the small signal approximntion.

Table I showg that is indeed the case. The firs column contains the amplitude of the external voltage $V_{e x t}$ changed in ateps from $1.05 \mathrm{kV}$ to $2.00 \mathrm{KV}$. Next column contsios, the gap voltege $\nabla_{S C}$ in $\mathrm{kV}$ as found from selfconsiatent solution $(6.2)$ for the Gaussian distribution iu initial clectron velocities. The third columa gives the small aignal approutmation gap voltage $V_{\text {so }}$ (in $\mathrm{kV}$ ). The lest column gives the phase ahift of tho gap voltage $\varphi_{a c}$ in repect to the applied external electric field. These values should be compared to the small signal approximation phase shift pusa which does not depend on the external amplitade. Table 2 summarizes the parameters of the cavity and the beam, used in these calculations. $V_{0}$ is the de gun voltage, $I_{0}$ is the do beam 
current and $\sigma$ is the rms velocity sprend of the incoming benm. $Q_{L}$ and $f_{L}$ are the beam londed parametera of the cavity found from equatious (7.20) and (7.21).

Table 3 illustrates the dependeret of the gap voltage $V_{s c}$ and its phase $\varphi_{\Delta e}$ on $\sigma$ of the initial Gaussian distribution. For $\sigma$ smaller than $10^{6} \mathrm{~m} /$ aec the result is the same as for zero spread velocity beam $\left(\sim \delta\left(v-v_{0}\right)\right)$.

Figure 1 presents the normalized velocity distribution of the beam as function of the dimensisnless velocity $(\mathbf{6 . 5})$ at the envity exit for different values of the of phase $\tau$ (6,6).

Figures 2.4 are the phage plots of $u / k$ versus $x / k$ for $k=0,2,0.25$ and 0.33 respectively.

Figure 5 rupresents the amplitude and the phase of the first harmonic of the beam current as lunclions of the dinensionless di:tance $x$ (B.13) inside the gap. Both the amplitude and the phase agree witb the result (8.18) obtained in the narrow gap npproximation.

\section{DISCLAIMER}

This report was prepared as an atcount of work sponsored by an agency of the United States Government. Neither the United Stales Goverament nor any ugency thereof, nor any of theit employees, makes any warranty, express or implied, or assumes any legal liability or responsj. bility for the aceurncy, completeness, ne uselulness of any information, apparatus, product, or process lisclosed, ot represents that its use would not infringe privately owned rights. Referenee herein to any specific commercial product, process, or service by ifade name, itademark, manufacturer, or otherwise does not necessurily constilute or imply its endorsement, recommendation, or lavoring by the United States Government or any agency theseof. The vit ivs and opinions of uuthors expressed herein do not necessarily state or reflect those of the Uniled States Govermment or any agency thereof. 


\section{Thble 1}

and

$\mathbf{V}_{\text {ext }}$ $p_{104}=-0.128490 D+00$

$\because n, V_{\text {oxt }}$

(n)

$0.105000 \mathrm{D}+04 \quad 0.851658 \mathrm{D}+03 \quad 0.851064 \mathrm{D}+03$ $0.110000 \mathrm{D}+04^{\prime} 0.802212 \mathrm{D}+03 \times 0.802220 \mathrm{D}+03$ 0.1150000 d $+04: 0.939760 \mathrm{D}+03$ $0.032775 \mathrm{D}+03$ $0.120000 \mathrm{D}+04 \ldots 0.0793921 \mathrm{D}+03$ $0.125000 \mathrm{D}+04: 0.101837 \mathrm{D}+04$ $0.073331 \mathrm{D}+03$

$0.101389 \mathrm{D}+03$

$0.105444 \mathrm{D}+04$

$0.109500 \mathrm{D}+04$ $0.135000 D+04 \cdot 0.108498 D+04$ $0.113556 \mathrm{D}+04$ $0.140000 \mathrm{D}+04$ $0.113554 D+04$ $0.1450000+04$ $0.117009 \mathrm{D}+04$ $0.1500000+04$

$0.121864 \mathrm{D}+04$ $0.1170110+04$

$0.121860 \mathrm{D}+04$

$0.1550000 \mathrm{D}+04$ $0.1257200 \mathrm{D}+04$

$0.125722 \mathrm{D}+04$

$0.120777 \mathrm{D}+04$ $0.160000 \mathrm{D}+04$

$0.120775 \mathrm{D}+04$

$0.165000 \mathrm{D}+04$

$0.133830 \mathrm{D}+04$

$0.170000 \mathrm{D}+04$

$0.137886 \mathrm{D}+04$

$0.133833 \mathrm{D}+04$

$0.13788 \mathrm{D}+04$

$-0.122439 D+00$ $-0.122433 D+\infty$ $-0.122433 \mathrm{D}+\infty$ $-0.122434 \mathrm{D}+\infty 0$ $-0.122434 \mathrm{D}+00$ $-0.122434 \mathrm{D}+00$ $-0.122435 D+\infty 0$ $-0.122435 \mathrm{D}+\infty 0$ $-0.122438 \mathrm{D}+\infty$ $0.175000 D+04$ 0.141941D + 04 $0.141944 \mathrm{D}+04$ $-0.122436 \mathrm{D}+\infty$ $-0.122438 \mathrm{D}+\infty 0$ $-0.122437 \mathrm{D}+00$ $-0,122437 \mathrm{D}+00$ $-0.122438 \mathrm{D}+09$ $0.1800000+04$ $0.145896 \mathrm{D}+04$

$0.146000 \mathrm{D}+04$ $-0.122438 D+00$ $0.1850000+04$ $0.150052 \mathrm{D}+04$ $0.150065 \mathrm{D}+04$ $0.180000 \mathrm{D}+04$ $0.154107 \mathrm{D}+04$ $0.154111 \mathrm{D}+04$ $-0.122430 D+\infty 0$ $0.105000 \mathrm{D}+04$ $0.158162 \mathrm{D}+04$ $0.200000 \mathrm{D}+04$ $0.162217 \mathrm{D}+.04$ $-0.122430 D+00$ $-0.122440 D+00$ $-0.122440 D+00$ $0.162222 \mathrm{D}+04$

$-0.122441 D+00$ 


\section{Tuble 2}

length $=0.016000 \mathrm{~m}$

$Q_{L}=040.48001$

$Q=1000.0$

$\mathrm{R} / \mathrm{Q}=85.0 \mathrm{Ohm}$

$f_{L}=0.35313 \mathrm{D}+09 \mathrm{~Hz}$

$f=0.3532 \mathrm{D}+09 \mathrm{~Hz}$

$\Delta f=0.20 \mathrm{D}+08 \mathrm{~Hz}$

$\mathrm{eV}_{0}=0.620000 \mathrm{D}+05 \mathrm{eV}$

$I_{0}=11.50 \mathrm{~A}$

$\because \sigma=0.13000+06 \mathrm{~m} / \mathrm{sec}$ 
Table 3

$\begin{array}{ccc}\sigma[\mathrm{m} / \mathrm{Bec}] & V_{o e}[\mathrm{kV}] & \varphi_{\text {se }}[\mathrm{rad}] \\ -10^{2} & 1.02217 & -0.122441 \\ 10^{3} & 1.02217 & -0.122441 \\ 10^{4} & 1.62217 & -0.122441 \\ 10^{8} & 1.62217 & -0.122441 \\ 10^{6} & 1.62212 & -0.122453 \\ 10^{7} & 1.61656 & -0.123648 \\ 1.4 \cdot 10^{7} & 1.61088 & -0.124835 \\ 1.8 \cdot 10^{7} & 1.60270 & -0.126468\end{array}$




\section{Concluaions}

The approach suggested in this work proves to be correct. The results obtajned agrec to a great accuracy with the small signal approximntion. In the limil of aerrow gap the solution gives reasonable results both for the amplitude and phase of the resonant harmonic of the beam current.

The next questions which should be addressed are how useful and how convenient is the Vasov appronch in general and with reapect to the klystron problem in particular. The calculation of the particle distribution aloag the klystron tube si uss to be atraight forward although substantial work need to be done.

Nevertheless, the approach looks promising. One can attempt to develop a onedimensional model of a kJystron which will include all important physies of the beam dynamics in multicavity system, including the interaction with the output cavity and crossover of the electron trajectories. The model takes into account the space charge effects in the cavities. The debunching effect of the space charge in drift seetions of the klyatron can be evaluated in pertvrbative manner using the ballistic approximation as the unperturbed solntion. Such a model might be nsefull as a fast and convenient ton for the klystron dosign. It can also provide information (at least as the first guesu) on the amplitudes and the phases of the gap voltages for klystron cavities. That might be useful as the input for numerical models of a klystron such as MASK.

Further work is needed to extend the pregent formulation into the region of relntivistic velocities.

\section{Acknowledgements}

The authora are grateful to all members of the Numerical Analysis Group for the joterest and stimulating discussions of the problem. Our specin gralitude goes to $\mathbf{B}$. Hertmannsfeld and $P$. Wilson for the encouragement nnd telp in our work. 


\section{References}

1. Vlasor, A. A., "On the Kinetic Theory of an Assembly of Particles with Collective Interaction." J. Phys. (U.S.S.R.) Q, 25 (19.55).

2. See, for example, N. A Krall and A. W. Thivelpiece, "Principles of Plasma $\therefore$ Physice," MeGraw-Hill (1973).

3. T. H. Stix, "The Theory of Plasme Waves," McGraw-Hin (1962).

4. G. M. Branch, Jre, IRE Traus. on Electron Devices, ED-8,\# 3, May 1061, p. 103.

5. M. Chodorow and O. Susskind, "Fundamentals of Microwave Electronics," MeGraw-Hill Company. 


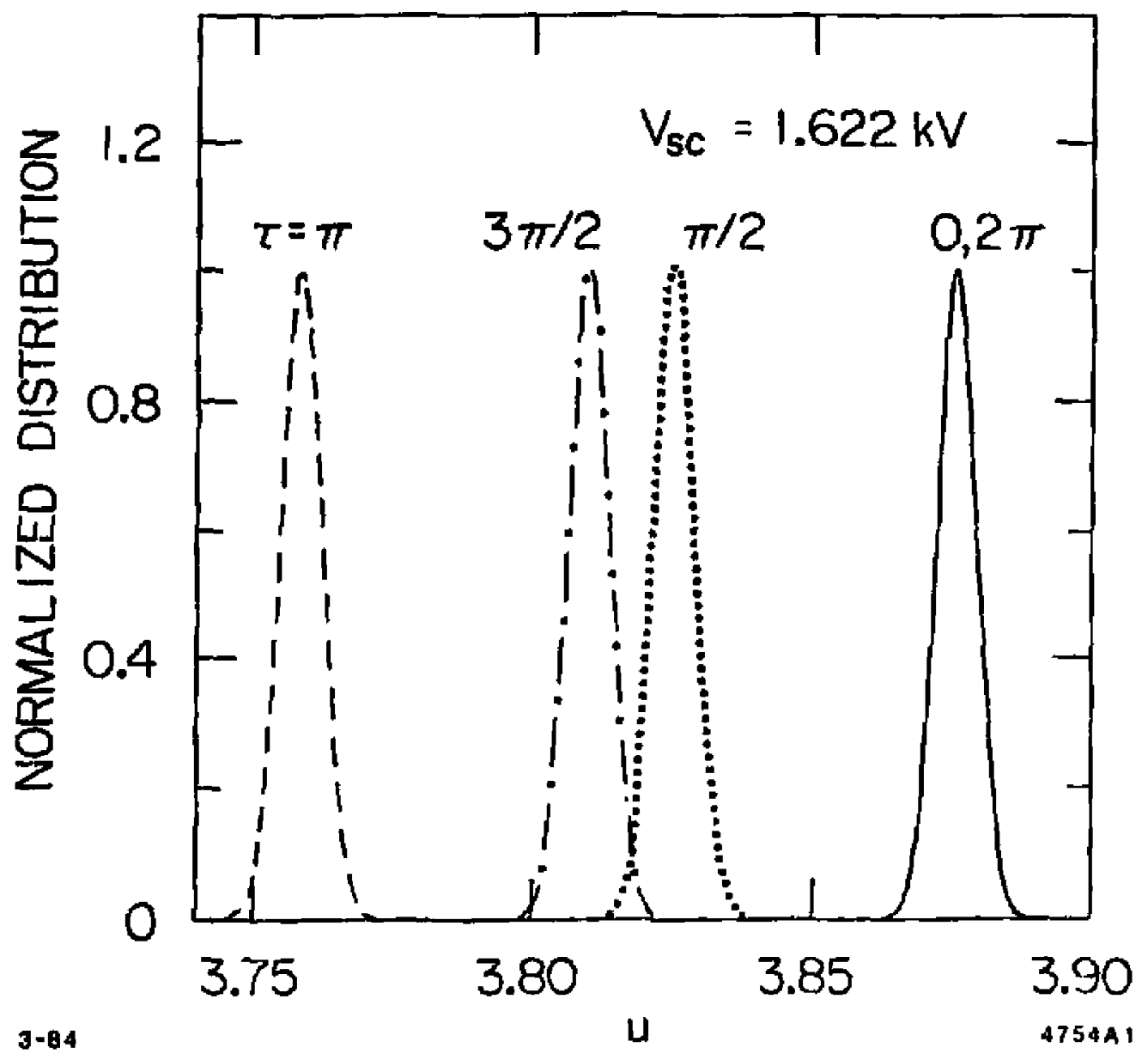

Fig. I 


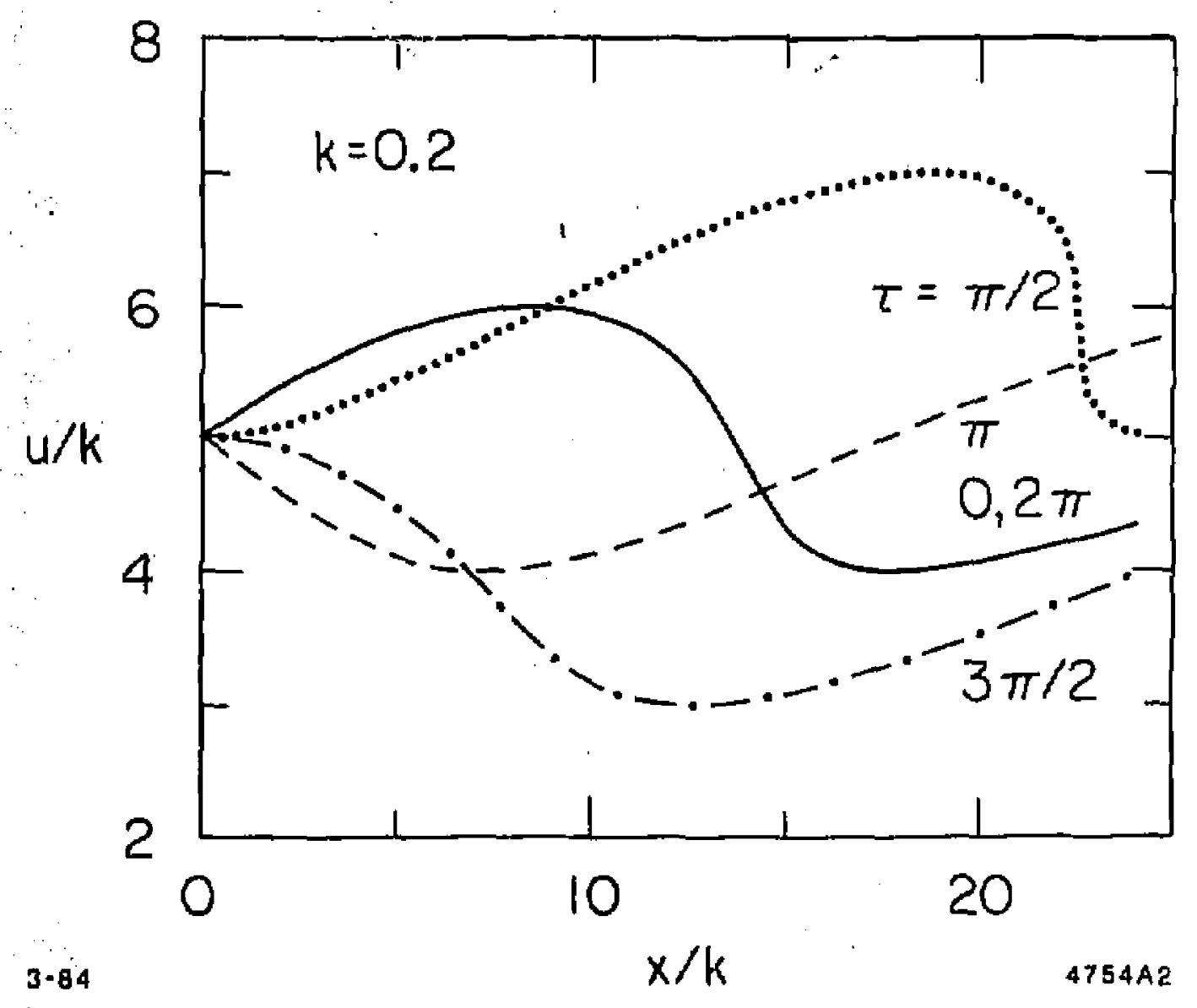

Fig. 2 


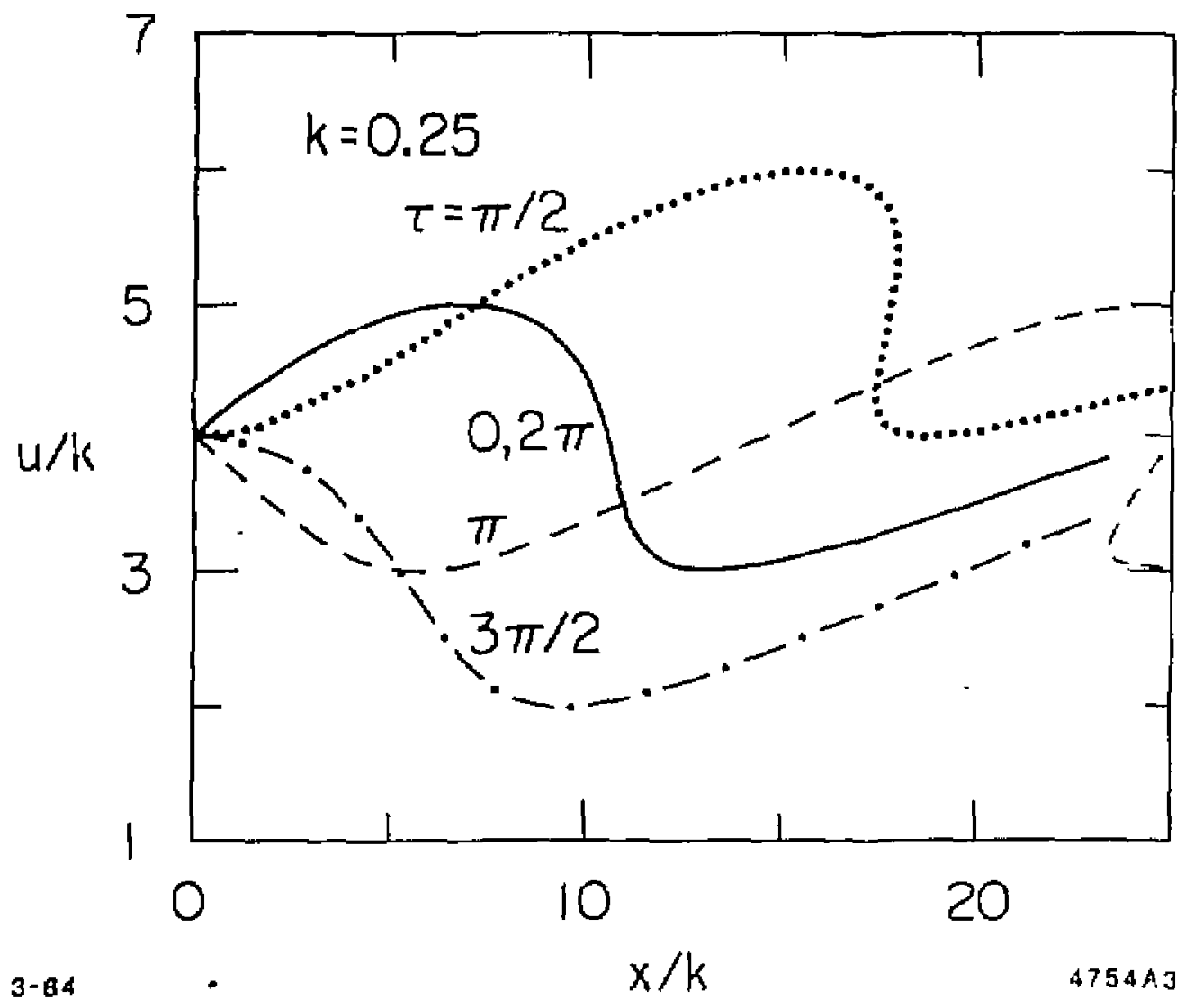

Fig. 3 
:

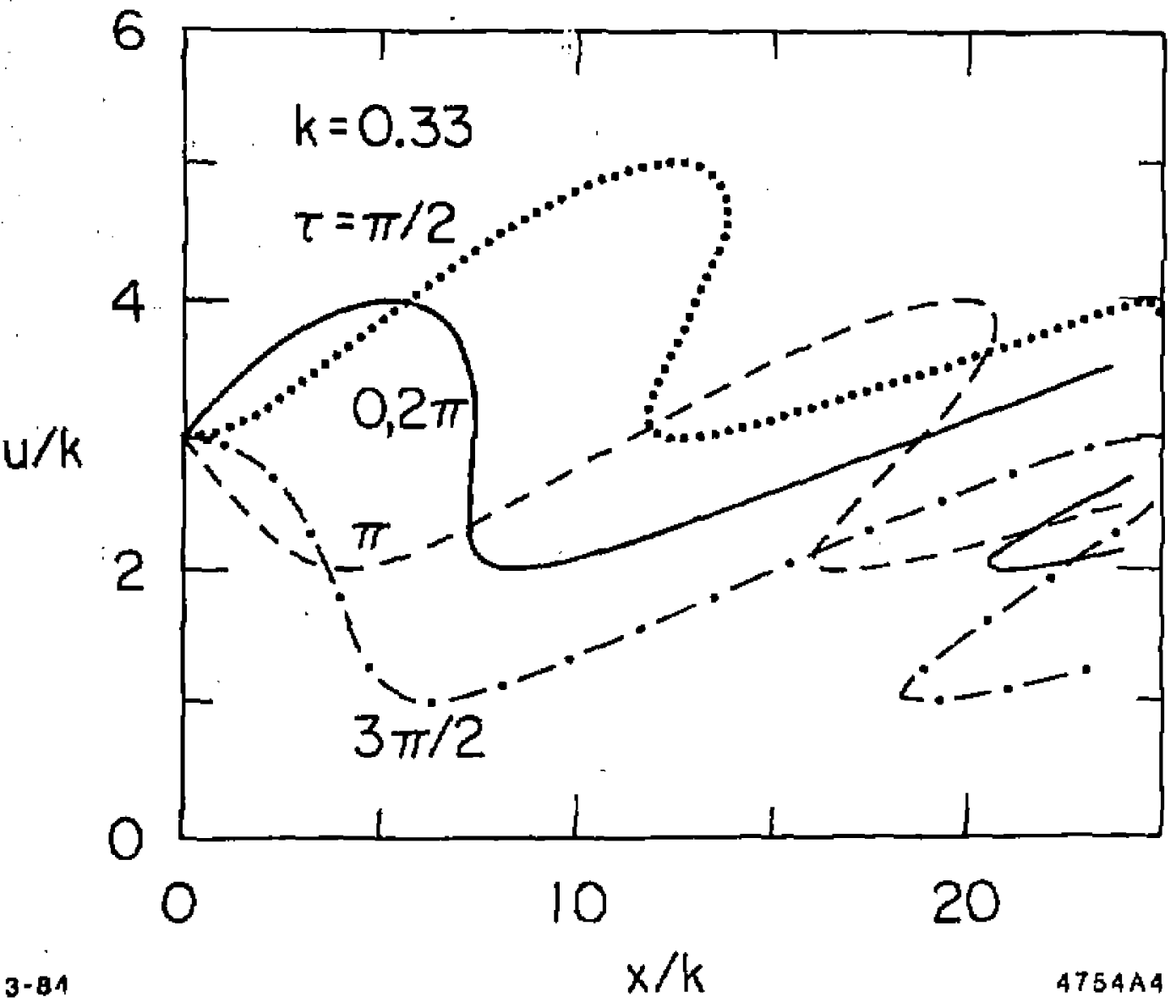

Fig. 4 


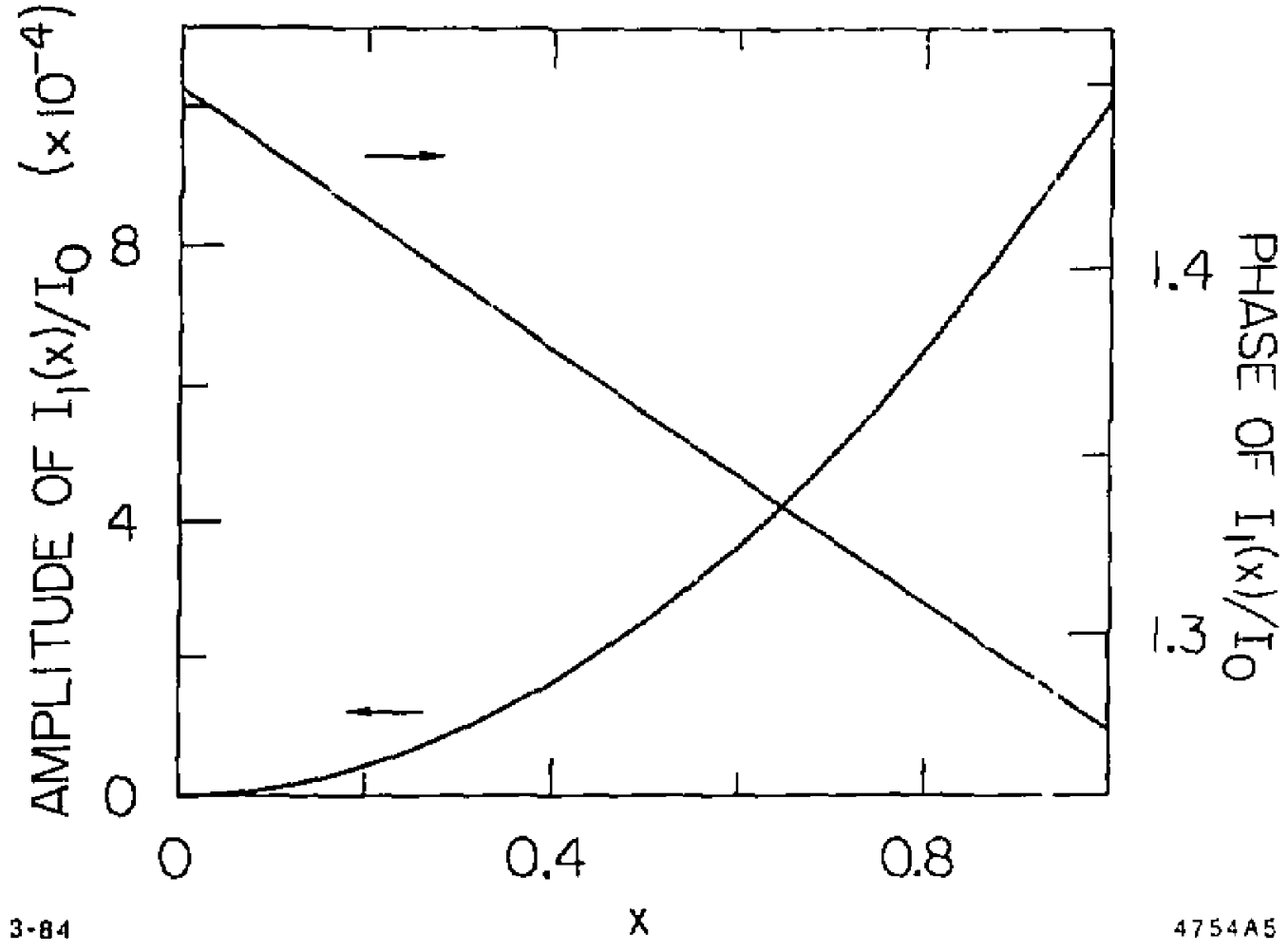

Fig. 5 